\title{
Image calibration and registration in cone-beam computed tomogram for measuring the accuracy of computer- aided implant surgery
}

Walter Y. H. Lam, Henry Y. T. Ngan, Peter Y. P. Wat, Henry W. K. Luk, Tazuko K. Goto, et al.

Walter Y. H. Lam, Henry Y. T. Ngan, Peter Y. P. Wat, Henry W. K. Luk, Tazuko K. Goto, Edmond H. N. Pow, "Image calibration and registration in cone-beam computed tomogram for measuring the accuracy of computeraided implant surgery," Proc. SPIE 9405, Image Processing: Machine Vision Applications VIII, 94050A (27 February 2015); doi: 10.1117/12.2083341

SPIE. Event: SPIE/IS\&T Electronic Imaging, 2015, San Francisco, California, United 


\title{
Image calibration and registration in cone-beam computed tomogram for measuring the accuracy of computer-aided implant surgery
}

\author{
Walter Y.H. Lam ${ }^{\mathrm{a}}$, Henry Y.T. Ngan ${ }^{\mathrm{b}}$, Peter Y.P. Wat ${ }^{\mathrm{a}}$, Henry W.K. Luk ${ }^{\mathrm{a}}$, \\ Tazuko K. Goto ${ }^{\text {a }}$, Edmond H.N. Pow ${ }^{\text {a* }}$ \\ aFaculty of Dentistry, The University of Hong Kong, Pokfulam, Hong Kong, China \\ ${ }^{b}$ Department of Mathematics, Hong Kong Baptist University, Kowloon Tong, Hong Kong, China
}

\begin{abstract}
Medical radiography is the use of radiation to "see through" a human body without breaching its integrity (surface). With computed tomography (CT)/cone beam computed tomography (CBCT), three-dimensional (3D) imaging can be produced. These imagings not only facilitate disease diagnosis but also enable computer-aided surgical planning/navigation. In dentistry, the common method for transfer of the virtual surgical planning to the patient (reality) is the use of surgical stent either with a preloaded planning (static) like a channel or facilitates real time surgical navigation (dynamic) after registration with fiducial markers (RF). This paper describes using the corner of a cube as a radiopaque fiducial marker on an acrylic (plastic) stent, this RF allows robust calibration and registration of Cartesian (x, y, z)-coordinates for linking up the patient (reality) and the imaging (virtuality) and hence the surgical planning can be transferred in either static or dynamic way. The accuracy of computer-aided implant surgery was measured with reference to coordinates. In our preliminary model surgery, a dental implant was planned virtually and placed with preloaded surgical guide. The deviation of the placed implant apex from the planning was $\mathrm{x}=+0.56 \mathrm{~mm}$ (more right), $\mathrm{y}=-0.05 \mathrm{~mm}$ (deeper), $\mathrm{z}=-0.26 \mathrm{~mm}$ (more lingual) which was within clinically $2 \mathrm{~mm}$ safety range. For comparison with the virtual planning, the physically placed implant was CT/CBCT scanned and errors may be introduced. The difference of the actual implant apex to the virtual apex was $\mathrm{x}=0.00 \mathrm{~mm}, \mathrm{y}=+0.21 \mathrm{~mm}$ (shallower), $\mathrm{z}=-1.35 \mathrm{~mm}$ (more lingual) and this should be brought in mind when interpret the results.
\end{abstract}

Keywords: Cone beam computed tomography, Cartesian coordinates, implant surgical guide, mapping, rigid registration

\section{INTRODUCTION}

Medical radiography is the use of electromagnetic radiation, most commonly the X-rays, to view the internal structure of a human body without breaching its surface integrity. Human structures absorb and therefore attenuate radiation in different levels with respect to their specific tissue density (radiopacity). Bone and teeth are mineralized tissues rich in calcium ions of high atomic number, they appear white in the imaging since most of the radiations were attenuated and the detector was little exposed. Meanwhile the soft tissues composed of mainly water and the air cavities which do not attenuate the radiation much and cause extensive exposure leading to dark imaging. In the past, when only plain radiographs were available, the acquired radiographic images represented summative radiation attenuation by the tissues between the source and the detector. While plain radiography is useful for diagnosis of simple disease e.g. bone fracture of limbs, its application might be limited when multi-layered composite structures such as abdomen or head are involved which makes the interpretation of images extremely difficult.

With the advancement of digital technology, in CT/CBCT, tissues along the radiation path can be differentiated and reconstruct into the gray-scale three-dimensional (3D) image by shaded surface display (SSD) and volume rendering as well as the three orthogonal views in axial, coronal and sagittal by multi-planar reformatting (MPR). These were based on a series of plain radiographs taken from different levels in CTs or from different angulations in CBCTs. CBCTs require much lower radiation dosage to the patient in single rotation of the $\mathrm{X}$-ray source/detector assemble in comparison of multiple rotations in CTs. These imagings ${ }^{1,2}$ are accurate representation of the real object as verified by linear and three dimensional measurements. This enables computer-aided surgical planning, e.g. joint replacement in the orthopedics and accurate placement of oral implants in the dentistry. The surgeon may perform the trial surgery virtually or on a rapid prototyped stereolithographic (SLG) model and plan for reconstruction of the surgical defect.

Image Processing: Machine Vision Applications VIII, edited by Edmund Y. Lam, Kurt S. Niel,

Proc. of SPIE-IS\&T Electronic Imaging, SPIE Vol. 9405, 94050A · c) 2015 SPIE-IS\&T

CCC code: $0277-786 X / 15 / \$ 18 \cdot$ doi: $10.1117 / 12.000000$ 
To transfer the virtually planned implant position to the patient (reality), a precise registration between the virtuality and reality is needed. In orthopedic, surgeons register specific points on the bone to match the actual anatomy to the virtual model during surgery. In dentistry, patients usually need to wear a plastic stent with radiopaque markers seating on the teeth which are stable and reproducible during the imaging acquisition. These markers are either simply providing a intended final outcome (future tooth crown shape) for planning or a piece of surgical information in the form of channel showing the path of drilling (static guidance) ${ }^{3}$. Alternatively, the markers may help relating the imaging to the patient (usually by metal spheres) for real-time navigation (dynamic guidance) ${ }^{4}$. In this paper, the corner of a cube acts as a fiducial marker (RF) which allows a robust calibration and registration of Cartesian (x, y, z)-coordinates for both the patient and the imaging while they are being linked up, the surgical planning can then be transferred in either way. The final surgical result can easily be compared to the planning with reference to the difference in $(\mathrm{x}, \mathrm{y}, \mathrm{z})$-coordinates.

This paper is organized as follows. Section 2 gives a review of current implant surgical guidance in dentistry. Section 3 presents the mathematics and procedure of the planning and actual surgical result. Section 4 offers the details of the experiment such as material and method, and the corresponding results. Lastly, Section 5 draws the conclusion.

\section{REVIEW OF CURRENT IMPLANT SURGICAL GUIDANCE IN DENTISTRY}

Titanium oral implants are a novel treatment modality for replacement of missing teeth. It is a biocompatible screw-like metal cylinder first surgically inserted and then integrated in human jaw bone via a process named osseo-integration. The missing tooth can then be replaced by attaching a prosthetic crown to the integrated implant. Dental implant has various diameters and lengths available and its selection depends mainly on the volume of bone and any intervening vital anatomical structures such as the nerve trunk. In addition, the position and angulation of the implant is also crucial for the fabricating as well as the long term success of the prosthesis.

Traditionally, a plastic/wax tooth crown is physically set on the stone model of teeth in harmony with adjacent/opposing teeth and the surgery is planned accordingly. The entry point $\left(\mathrm{A}_{\mathrm{r}}\right.$ in Figure 1$)$ and the angulation of an implant (O-O' in Figure 1) is backward planned with reference to the future tooth crown (final outcome). The surgical planning in the form of a channel will be carried by a stent with radiopaque marker (radiographic stent) and verified by taking a CT/CBCT. Any changes to the original plan can be calculated with a simple geometric algorithm and the stent (now become a surgical stent) can then be modified with mechanical positioning devices or drilling machines to provide surgical plan to the surgeons (new entry point $\mathrm{B}_{\mathrm{r}}$ and new angulation N-N' in Figure 1$)^{3}$.

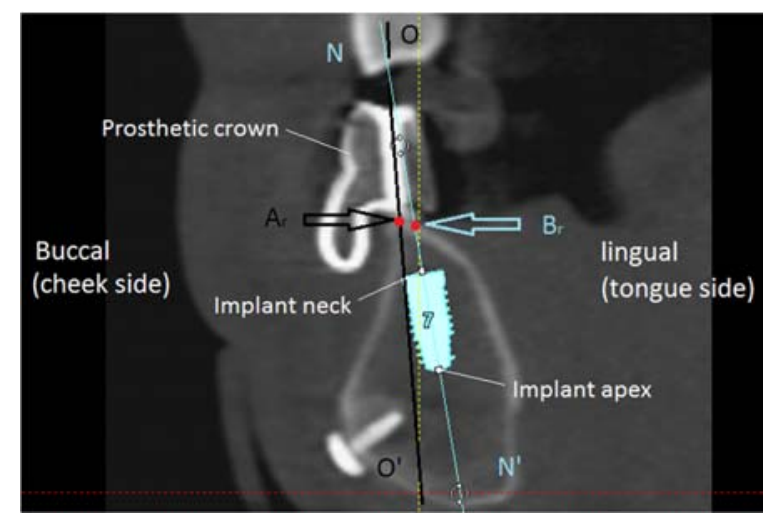

Figure 1. A cross-sectional view of a CBCT taken for implant planning. Outline of the future tooth crown and the preplanned channel was lined by a radiopaque material gutta percha. Original plan pass the center of future tooth crown but not the center of bone. A change of implant's angulation and entry point was therefore decided. $A_{r}$ is the pre-CT implant entry point and $\mathrm{B}_{\mathrm{r}}$ is the post-CT entry point; axis $\mathrm{O}-\mathrm{O}$ ' is the pre-CT implant long axis and N-N' is the post-CT implant long axis.

Rapid prototyped SLG surgical guide may be used to transfer the virtual implant surgical planning back to the patient ${ }^{5}$. For single or short span missing teeth, radiographic stent may be omitted since adjacent teeth may provide information to set a virtual tooth crown. For multiple missing teeth, the information of future tooth crowns may be provided by wearing 
a radiographic stent during $\mathrm{CT} / \mathrm{CBCT}$ acquisition. Alternatively, patient's existing plastic denture (which replaces missing teeth) seated on the stone teeth model may be digitalized (e.g. by contact scanning) and fused to patient's CT/CBCT.

Real-time surgical navigation with reference to the CT/CBCT imaging is another computer-aided surgical option. Navigation has been used extensively in the orthopedics for hip and knee joint replacement, in which the surface and the functional movement of the joint needs to be captured on the operation table with a tracking system attached directly to the bone ${ }^{6}$. In dentistry, dynamic guidance has advantage over the static channel-type surgical guide which allows the surgeons to make adjustment to the planned position during surgery. Stents with radiopaque markers should be worn by the patient during image acquisition. These markers were then registered on both imaging and patient using point-to-point ${ }^{7}$ or pixel registration ${ }^{8}$. The surgical drilling instrument was real-time tracked and linked with the imaging. Finally, surgical guidance may be provided in the form of augmented reality. Head mounted display (HMD) ${ }^{9}$ can project the virtual plan into the real optical path by photogrammetric calibrating the coordinates of the reality and virtuality. While both navigation and stent guidance allow precise position of dental implants, stent-based implant guidance were more popular in private practices since the clinicians only need the stent and outsource complex procedures to the manufacturers. In institutions, the navigation was favored since this can be used in many cranio-maxillofacial procedure like removal of tumor, tissue sampling (biopsies) and temporomandibular joint (TMJ) surgery (arthroscopy).

Errors could be accumulated from the image acquisition, the registration, the adherence to and/or degree of freedom of the guidance 4 . The precision of implant surgery can be assessed by comparing the position and angulation of the placed implant with the planned one. Implant apex (Figure 1) is the part of an implant that is most far away from the entry point and it was suggested to be most deviated from the planning and was therefore measured when assess transfer error. The placed implant (in either in vitro teeth models or in vivo patients) will be CT/CBCT scanned and superimposed with the virtual planning (image fusion), their relative difference would demonstrate the accuracy of guidance ${ }^{10}$. This manipulation requires specialized software for registration and matching however this result is difficult to validate physically.

\section{ROBUST IMAGE CALIBRATION AND REGISTRATION OF THE PLANNING AND ACTUAL SURGICAL RESULT}

This section describes the mathematics of robust image calibration and registration of the planning and actual surgical result. A computer-aided implant surgery model is shown in Fig. 2.

\subsection{Cartesian coordinate in the object (pre-operative; real physical domain)}

The corner of a cube of the surgical stent was chosen to be the origin $\left(0_{x}, 0_{y}, 0_{z}\right)$ and the three orthogonal surfaces forming this corner represent the $\mathrm{x}-, \mathrm{y}-$ and $\mathrm{z}-$ planes. Where two planes intersect, these represent the axis. Mathematically, the $(x, y, z)$-coordinates of object $i$ can be expressed as

$$
O_{i}=\left(x_{i}, y_{i}, z_{i}\right)
$$

3.2 Cartesian coordinate in the virtual imaging/surgical planning (pre-operative; computerized virtual domain) The corner of a cube of the surgical stent in the imaging was chosen to be the origin $\left(0_{\mathrm{x}}, 0_{\mathrm{y}}, 0_{\mathrm{z}}\right)$. Mathematically, the $(x, y, z)$-coordinates of computerized object $i$ can be expressed as

$$
C_{i}=\left(X_{i}, Y_{i}, Z_{i}\right)
$$

\subsection{Rigid registration of the virtual imaging to physical object (Image-to-physical (IP) transformation) $F\left(w_{i}\right)=W_{i}$}

where $w_{i} \in\left(x_{i}, y_{i}, z_{i}\right)$ and $W_{i} \in\left(X_{i}, Y_{i}, Z_{i}\right)$ and $\mathrm{F}: O \rightarrow C$ is a rigid transformation for real objects $i, j$ to the computerized objects $i, j$ in the CBCT. F has an isometry property of preserving $\mathrm{d}\left(F\left(w_{i}\right), F\left(w_{j}\right)\right)=d\left(W_{i}, W_{j}\right)$ and also has no reflection which preserves the space orientation. 
In the computerized virtual domain, virtual implant was planned with the coordinates of virtual implant apex and neck which are denoted as $C_{i a}=\left(X_{i a}, Y_{i a}, Z_{i a}\right)$ and $C_{i n}=\left(X_{i n}, Y_{i n}, Z_{i n}\right)$, respectively. After implant placement with the aid of surgical stent/navigation, the coordinates of the actual implant apex and neck were $O_{i a}=\left(X_{i a}, Y_{i a}, Z_{i a}\right)$ and $O_{i n}=\left(X_{i n}, Y_{i n}\right.$, $Z_{\text {in }}$,respectively.

Ideally $O_{i a}$ and $O_{i n}$ should have same coordinates as $C_{i a}$ and $C_{i n}$ respectively, in the absence of any transformation and transfer error, i.e.,

$$
C_{i}=\left(X_{i}, Y_{i}, Z_{i}\right)=\left(x_{i}, y_{i}, z_{i}\right)=O_{i}
$$

To measure the precision of computer aided implant surgery, the implant apex/neck coordinates of the post-operative real physical domain (actual placement) $O_{i a}$ and $O_{i n}$ and the pre-operative computerized virtual domain (virtual planning) $C_{i a}$ and $C_{i n}$ were compared. Coordinate measuring machine (CMM) may be used to measure the coordinate of a physical domain. This is possible with the corner of the cube which acts as the origin. However, this is not practical in clinical cases since the implant apex is enclosed by tissues. Another approach is to transfer the post-operative real physical domain to post-operative computerized virtual domain by taking the CT/CBCT of the object (stone model or patient) which again involves the registration of the virtual imaging to the physical object (IP transformation).

The virtual pre-operative (planning) and post-operative (after treatment) domains were then registered (Image-to-image (II) transformation) for superimposition of the planned and the placed implant. The II transformation was based on various algorithms of matching reference points, surfaces or pixels of the imagings. Alternatively, the coordinates of the implant, either in the physical or virtual domains, may be measured with reference to a common fixed reference point origin $O$ (in this paper the corner of a cube). The virtual measurement of coordinates involves matrix transformation (rotation) of the imaging so the horizontal and vertical axes of the imaging fit to the corner of cube.

For our corner of the cube $O_{i}$, let $v_{i}=\left[\begin{array}{l}x_{i} \\ y_{i} \\ z_{i}\end{array}\right]$ be a basis vector of 0 , such that there exists an affine transformation $F_{a f f i n e}$ : $O \rightarrow C$ for translation, scaling, rotation and shears to a new computerized object $C_{i}$. For example, a translation of our cub e object $O_{i}$ is expressed as

$$
\left[\begin{array}{l}
X_{i} \\
Y_{i} \\
Z_{i}
\end{array}\right]=\left[\begin{array}{l}
x_{i} \\
y_{i} \\
z_{i}
\end{array}\right]+\left[\begin{array}{l}
t_{x} \\
t_{y} \\
t_{z}
\end{array}\right]
$$

where $\left[\begin{array}{l}t_{x} \\ t_{y} \\ t_{z}\end{array}\right]$ is a translational factor $\left[\begin{array}{c}X_{i} \\ Y_{i} \\ Z_{i}\end{array}\right] \in C$.

A scaling of our cube $O_{i}$ is expressed as

$$
\left[\begin{array}{c}
X_{i} \\
Y_{i} \\
Z_{i} \\
1
\end{array}\right]=\left[\begin{array}{cccc}
S_{x} & 0 & 0 & 0 \\
0 & S_{y} & 0 & 0 \\
0 & 0 & S_{z} & 0 \\
0 & 0 & 0 & 1
\end{array}\right] *\left[\begin{array}{c}
x_{i} \\
y_{i} \\
Z_{i} \\
1
\end{array}\right]
$$

where $\left[\begin{array}{l}S_{x} \\ S_{y} \\ S_{z}\end{array}\right]$ is a scaling factor.

A rotation of our cube $O_{i}$ on the computerized domain $C_{i}=R_{j}(\alpha) \cdot\left[\begin{array}{c}x_{i} \\ y_{i} \\ z_{i} \\ 1\end{array}\right]$ for $j \in\{x, y, z\}$ direction, is expressed as 


$$
\begin{aligned}
& R_{x}(\alpha)=\left[\begin{array}{cccc}
1 & 0 & 0 & 0 \\
0 & c & -s & 0 \\
0 & s & c & 0 \\
0 & 0 & 0 & 1
\end{array}\right] \\
& R_{y}(\alpha)=\left[\begin{array}{cccc}
c & 0 & s & 0 \\
0 & 1 & 0 & 0 \\
-s & 0 & c & 0 \\
0 & 0 & 0 & 1
\end{array}\right] \\
& R_{z}(\alpha)=\left[\begin{array}{llll}
c & -S & 0 & 0 \\
S & c & 0 & 0 \\
0 & 0 & 1 & 0 \\
0 & 0 & 0 & 1
\end{array}\right]
\end{aligned}
$$

where $c=\cos (\alpha), s=\sin (\alpha)$ and $\alpha$ is the rotation angle.

The accuracy of transferring of virtual implant planning to surgery may be measured by the deviation of coordinate of the placed implant with virtually planned implant by root-square distance (Part I of the experiment). The coordinates of the placed implant may be obtained physically by CMM measurement or virtually by CT/CBCT. The accuracy of coordinate measurement may be measured by the deviation of coordinate of the virtualized implant with actual placed implant by root-square distance (Part II).

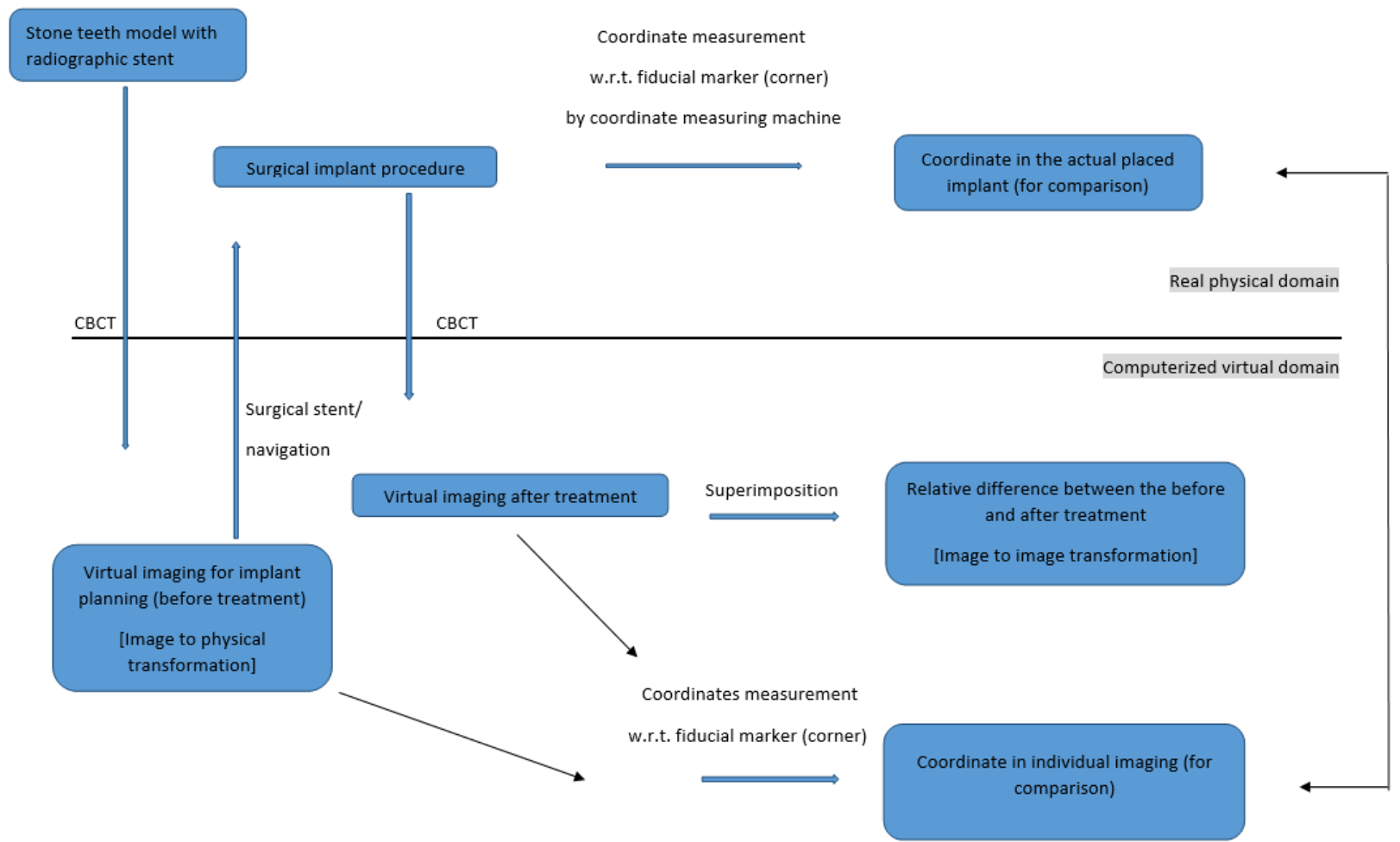

Figure 2. Flowchart showing the workflow of the computer aided implant surgery (Left hand side) and the methods for measuring its transfer error (Right hand side). 


\section{EXPERIMENT}

This section is all about the experiment. Section 4.1 gives the details of the material and method in the image calibration and registration, Section 4.2 offers the corresponding experimental results and Section 4.3 addresses some discussions about the results.

\subsection{Material and Method}

\subsubsection{Part I}

[Physical] A patient with missing maxillary (upper) left central incisor was recruited and alginate impressions (soft mould) of the maxillary and mandibular (lower) teeth were made. From these, upper and lower stone teeth models were poured. An acrylic (plastic) stent with a $4 \mathrm{~mm}$ diameter channel filled with a radiopaque gutta percha was fitted on the upper stone teeth model (Figure 2a) and a $1 \mathrm{~cm} \times 1 \mathrm{~cm} \times 1 \mathrm{~cm}$ cubic corner painted with radiopaque Barium Sulphate $\left(\mathrm{BaSO}_{4}\right)$ was incorporated.

[Virtual] Cone-beam computed tomogram (iCAT classic, USA; tube voltage $120 \mathrm{kVp}$, tube current $3-7 \mathrm{~mA}$ ) was taken (Figure 2b). Scan protocol was selected with the largest Field of view (FOV) and highest available resolution (i.e. full 13 $\mathrm{cm} 40$ seconds 0.25 voxel high resolution). Then, 599 frames of 2D planar images were acquired. The imaging was transferred from the iCAT workstation to a personal computer (Dell Precision T1700 workstation with a graphic card NVIDIA Quadro K2000 and a 23-inch Dell P2314H LED monitor with a screen resolution of $1920 \times 1080$ ) loaded with an implant planning software SimPlant Pro (Version 16.0, Materialise NV, Leuven, Belgium) in the format of digital imaging and communications in medicine (DICOM).

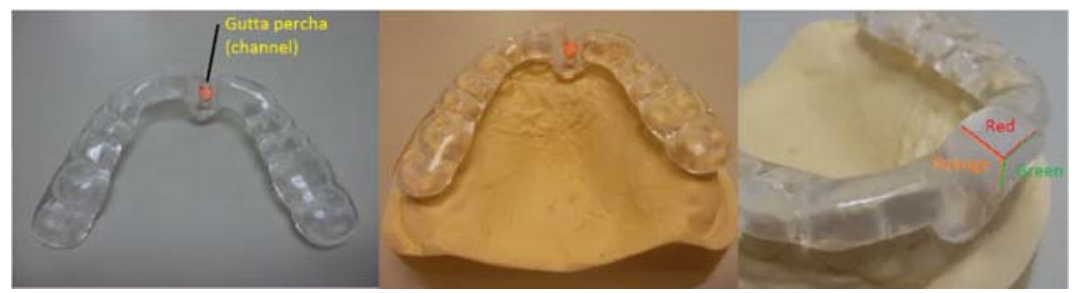

Figure 2a. Stone teeth model and acrylic stent. Corner of a cube on the stent defined the origin $O$ of both physical and virtual domains. Three colored orthogonal surfaces of the cube (orange, green and red) corresponding to respective windows in SimPlant Pro (see Figure 3a)

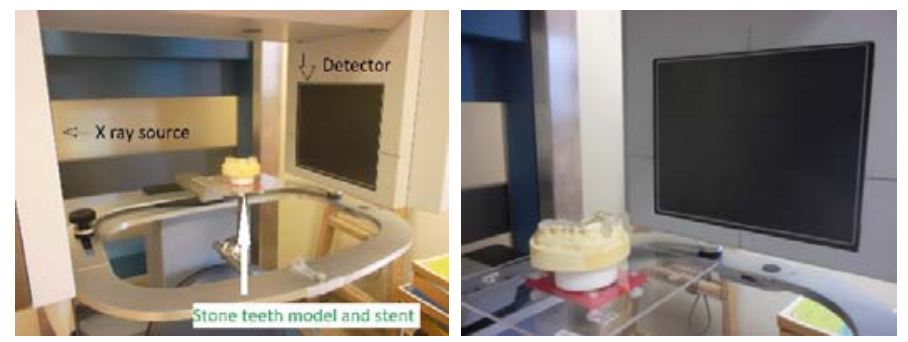

Figure $2 \mathrm{~b}$. The stone teeth model and the stent were loaded in the CBCT. The x-ray source and the detector moves simultaneously in one rotation during the imaging acquisition.
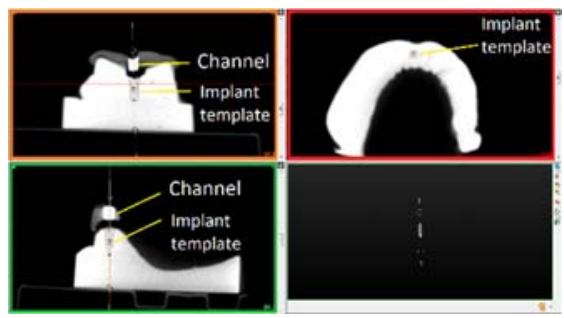
Figure 3a. Placement of the virtual implant template with a reference to the channel in the SimPlant Pro. Upper left: orange window; upper right: red window; lower left: green window; lower right: 3D virtual implant template and its imagining long axis.
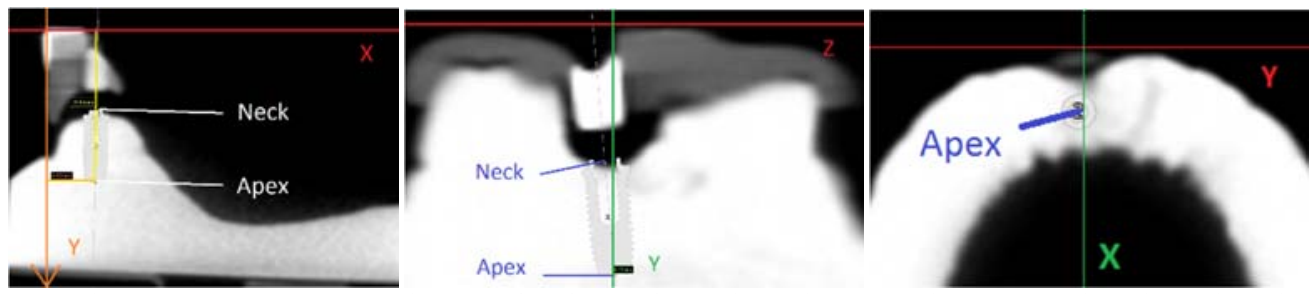

Figure $3 b$. Coordinate measurement of the apex/neck of planned implant template. Note that the fiducial marker (the corner) defined two axis lines and the origin $O$ where they meet.

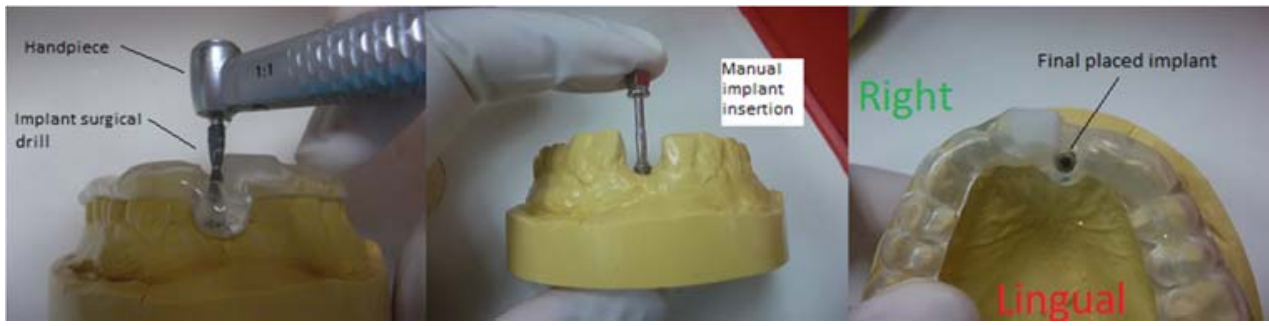

Figure 4a. Computer aided implant surgery with stent guidance on a stone teeth model. Left: Implant site preparation (soft bone drilling protocol $2 \mathrm{~mm}$ and 2.4/2.8mm drill) under the guidance of the stent's channel; Middle: Manual insertion of an implant into the prepared site; Right: Seating of the stent after implant placement shows the implant roughly placed following the channel of the stent.
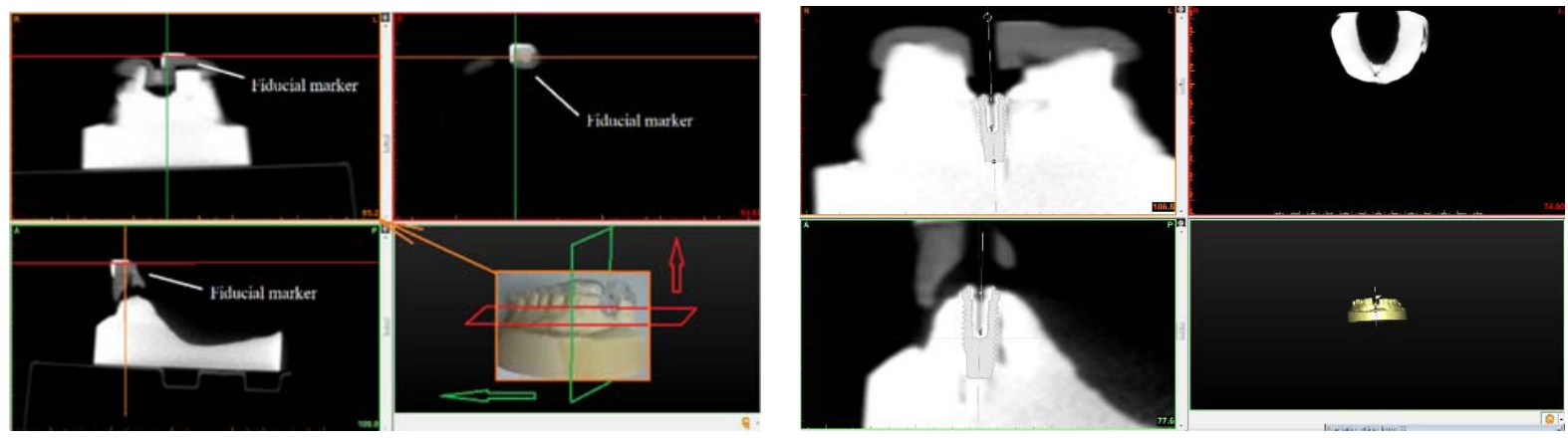

Figure 4b. Left: Post-operative CBCT (with the stent) and manual mapping the virtual axis lines to fiducial marker; Right: Precise locating of the implant apex was facilitated by manual mapping the implant template to the radiopacity of the placed implant in imaging.

[Virtual] In the SimPlant Pro software, dental implant (Brånemark system, $\varnothing 4.1 \mathrm{~mm} \times 13 \mathrm{~mm}$ [diameter $\mathrm{x}$ length], Nobel Biocare, Sweden) was selected with respect to the cross-sectional "bone" images. For the sake of simplicity in this preliminary study, no change in the entry point or angulation was made to the channel (Figure 3a). Corresponding implant template was superimposed onto the imaging. The corner of cube was manually registered to the virtual axis lines of the SimPlant Pro (which represent the position of two other orthogonal views). The (x,y, z)-coordinations for $C_{i}^{1}$ and $C_{i}^{2}$ of the virtual implant apex and neck, which are the tangential distances to the axe lines, were measured manually as gold standard (Figure $3 b)^{10}$.

[Physical] The gutta percha in the channel was removed and the model was prepared following the channel with $2 \mathrm{~mm}$ and 2.4/2.8mm drill. After removal of the stent, an implant was inserted into the drilled hole (osteotomy site) (Figure 4a).

[Virtual] Post-operative CBCT of the stone teeth model and the stent was taken using the same machine and scanning 
protocol. Corresponding implant template was fitted to the placed implant in the CBCT imaging to facilitate the location of implant apex. Manual registration was performed and the (x,y and $\mathrm{z}$ ) coordination $C_{i}^{2}$ of the implant apex and neck were measured with SimPlant Pro (Figure 4b).

A square root of distance between various two computerized objects $C_{i}^{1}$ and $C_{i}^{2}$ was calculated. The normalized cross correlation (NCC) will be measured in the future experiments.

\subsubsection{Part II}

[Physical] A duplicate of upper stone teeth model of the same patient was made. On this model, an implant (Brånemark system, $\varnothing 4.1 \mathrm{~mm} \times 13 \mathrm{~mm}$, Nobel Biocare, Sweden) was arbitrarily placed and the embedded implant apex was later exposed by removing the surrounding stone for measurement (Figure 5a). The coordinates of the implant apex was physically measured as a gold standard by a digital toolmaker's microscope with accuracy to $0.0025 \mathrm{~mm}$ (Leitz Wetzlar WM Standard Toolmakers' Microscope, Germany) with reference to the corner of cube (origin $O$ ) on the stent (Figure 5b). This microscope was functioned as a planar CMM. Acrylic platforms, parallel to the surfaces of the cube, were attached to the stone model with the aid of a parallelometer (Miko Parallelometer MP2000E, Metaux Preceus, Metalor, Switzerland). Planar coordinates was measured by the microscope with the stone model loaded parallel to one plane by the acrylic platform.

[Virtual] The duplicated model and the stent was CBCT scanned and its apex coordinates with reference to the corner of cube was measured in SimPlant Pro as described in Part I.

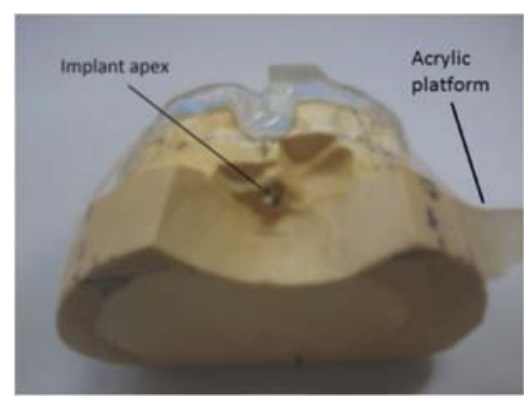

Figure 5a. Stone teeth model with exposed implant apex.

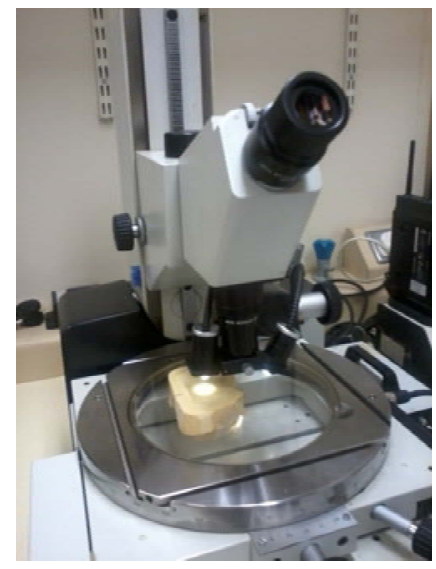

Figure 5b. Stone teeth model and the stent were loaded on the toolmaker's microscope for physically measuring the coordinates with reference to the origin $O$ on the stent. 


\subsection{Result \\ 4.2.1 Part I}

The $(\mathrm{x}, \mathrm{y}, \mathrm{z})$-coordination $C_{i}^{1}$ of the virtual implant apex was $\mathrm{x}=-0.19 \mathrm{~mm}, \mathrm{y}=-21.19 \mathrm{~mm}, \mathrm{z}=-6.05 \mathrm{~mm}$ and of the virtual implant neck was $\mathrm{x}=-1.18 \mathrm{~mm}, \mathrm{y}=-10.95 \mathrm{~mm}, \mathrm{z}=-6.29 \mathrm{~mm}$. The $(\mathrm{x}, \mathrm{y}, \mathrm{z})$-coordination $C_{i}^{2}$ of the actual placed implant apex with the preloaded surgical guide was $\mathrm{x}=+0.37 \mathrm{~mm}, \mathrm{y}=-21.24 \mathrm{~mm}, \mathrm{z}=-6.31 \mathrm{~mm}$ and of the actual implant neck was $\mathrm{x}=-$ $0.12 \mathrm{~mm}, \mathrm{y}=-11.03 \mathrm{~mm}, \mathrm{z}=-6.57 \mathrm{~mm}$.

The difference of the implant positions was $C_{i}^{2}-C_{i}^{1}$ (actual placed - virtual planned). Herein, for the implant apex the difference was $\mathrm{x}=0.56 \mathrm{~mm}$ (more right), $\mathrm{y}=-0.05 \mathrm{~mm}$ (deeper), $\mathrm{z}=-0.26 \mathrm{~mm}$ (more lingual) and for the implant neck the difference was $x=1.06 \mathrm{~mm}$ (more right), $y=0.01 \mathrm{~mm}$ (deeper), $\mathrm{z}=-0.28 \mathrm{~mm}$ (more lingual). The square root difference of the implant apex and implant neck were $0.62 \mathrm{~mm}$ (more right, deeper and more lingual) and 1.10mm (more right, deeper and more lingual) respectively.

\subsubsection{Part II}

The (x, y, z)-coordination $C_{i}^{1}$ of the virtual implant apex was $\mathrm{x}=-0.13 \mathrm{~mm}, \mathrm{y}=-23.84 \mathrm{~mm}, \mathrm{z}=-4.86 \mathrm{~mm}$ and the (x, $\left.\mathrm{y}, \mathrm{z}\right)$ coordination $C_{i}^{2}$ of the reality implant apex was $\mathrm{x}=-0.13 \mathrm{~mm}, \mathrm{y}=-23.63 \mathrm{~mm}, \mathrm{z}=-6.21 \mathrm{~mm}$. The difference of the implant apex was $C_{i}^{2}-C_{i}^{1}$ (actual placed - virtualize of the actual placed) and was $\mathrm{x}=0.00 \mathrm{~mm}, \mathrm{y}=+0.21 \mathrm{~mm}$ (shallower), $\mathrm{z}=-1.35 \mathrm{~mm}$ (more lingual). The square root difference of the implant apex was $1.36 \mathrm{~mm}$ (shallower and more lingual).

\subsection{Discussions}

In the first part of this study, acrylic surgical stent has shown a fairly accurate transfer of the virtual planning to the surgical field with respect to the actual placed implant apex in which it was $0.62 \mathrm{~mm}$ deviated from the planning. This is similar to the in vitro study by Besimo et al. ${ }^{11}$ which reported a transfer error of $0.6 \mathrm{~mm}(+/-0.4 \mathrm{~mm})$ when an implant placement on the maxilla (upper arch) was guided by a stent. The use of surgical stents in implant dentistry has a long history that a minimum additional equipment was needed. However, meticulous in every step from the image acquisition to the seating of the stent in patient's mouth are necessary since errors will accumulate and this guidance does not allow any intra-operative change in the surgical plan.

Surprisingly, the apex of the oral implant was more accurately placed then the implant neck which is in contrast to other studies $^{12}$. Possible explanation may be the surgical drill spin/rotate concentricity ${ }^{13}$. Specialized handpiece for an implant placement has a speed reducing 20 times (20:1) and higher torque (cutting efficiency) while the handpiece used in this study was not intended for the surgical purpose as it has a higher speed (1:1) and lower torque (Figure 4a). This would result in a different cutting mechanism ${ }^{14}$. The entry point of the stone model has received the longest drilling time, therefore it may be resulted in the greatest deviation. Moreover, the plastic stent material was soft and may allow a greater drilling deviation in comparison to the rigid metal. The relatively short $4 \mathrm{~mm}$ height of the channel provided by the plastic stent and the use of relatively smaller drill $\varnothing 2.4 / 2.8 \mathrm{~mm}$ in relation to this $4 \mathrm{~mm}$ channel may also leads to error in transfer. Most of the deviation of the placed implant was in the $\mathrm{x}$-axis (left and right) which may be due to the proximity of the RF cube to the channel. This RF cube may cause deviation of surgical instrument from the long axis of the channel. In our future study, a use of specialized implant handpiece, a better position of the RF cube, and a use of metal tube with an adequate length and diameter for guidance may reduce the transfer error seen in this study.

While there are many studies ${ }^{1,2,15,16,19}$ relating the virtual imaging of the skull to its physical reality, this paper is the first kind of study to our best knowledge that is relating the position of surgically placed dental implant in the virtual imaging to the physical reality. From this, the accuracy of the implant position in physical domain estimated by the computerized virtual domain can be determined. Image acquisition, registration, identification and measurement of the interested point (implant apex) will all contributes to an error in the virtual implant position. This would have a clinical implication since currently the transfer error of any guidance system was measured by the difference in the position of virtualized final implant position to that in the virtual planning. In the second part of this paper, the actual implant apex was $1.36 \mathrm{~mm}$ away from that in the imaging. Acquisition error ${ }^{15}$ can be as large as $0.27 \mathrm{~mm}$ or more in CBCT. An image-to-physical (IP) transformation ${ }^{16}$ has been suggested to have error of $0.5 \mathrm{~mm}$ in vitro. Target registration error (TRE) $)^{4,17,18}$, the "application accuracy" of the registration, measuring the distance between corresponding points other than the fiducial points after the registration. In our previous study ${ }^{10}$ using the same manual registration method on a cube, the TRE was $0.83 \mathrm{~mm}$ which 
was comparable to the study using a software automatic registration (mean $0.75 \mathrm{~mm})^{1}$. For an implant navigation system ${ }^{19}$ TRE has been found to be of $1.23 \mathrm{~mm}$ (average) and $1.87 \mathrm{~mm}$ (maximum) ${ }^{19}$.

The number of RF markers (fiducials) may have impact on the accuracy of registration. The more fiducials ${ }^{17}$ used in the registration the smaller TRE was anticipated. In this study, despite only one fiducial (corner of a cube) was used, this was matched three fiducials dimensionally in the SimPlant Pro and the TRE approached to that predicted for four fiducials $(1.48 \mathrm{~mm})$. For manual registration in SimPlant software, three virtual axis lines (x, y, $\mathrm{z}$ axes) were registered to the corresponding $(\mathrm{x}, \mathrm{y}, \mathrm{z})$ physical line angles of the cubic corner in three orthogonal views. In each orthogonal view, two axis lines were matched to one of the three 2D fiducial corners in these views (Figure 3b). Each axis line was therefore registered twice and this may explained the fairly accurate manual registration observed. Ideally, more (and well spread) fiducials should be used for more accurate registration particularly when automatic registration is available. However for manual registration, the thin horse-shoe shaped stent do not provide space for placement of extra "parallel" fiducial line angle to match with the axis lines in the sagittal plane (Figure $3 \mathrm{~b}$ plane X). Extra fiducial may be placed on another side of the channel and therefore provide a longer line angle of the fiducials to allow better registration with the axis lines in the coronal and transverse plane (Figure $3 \mathrm{~b}$ plane $\mathrm{Y}$ and $\mathrm{Z}$, Figure 6). In our previous study using the same manual registration method on an $8 \mathrm{~cm} \times 8 \mathrm{~cm} \times 4 \mathrm{~cm}$ cube, the TRE was less than the present study which may be due to the longer physical line angles for the registration with the virtual axis lines ${ }^{10}$.

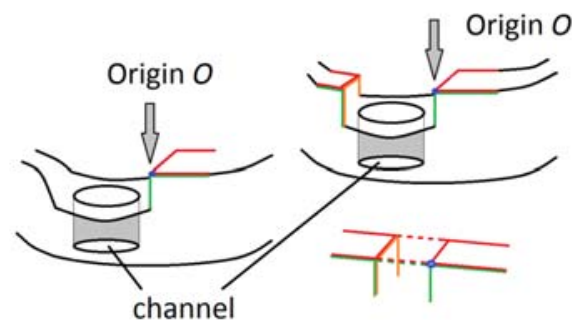

Figure 6. Cubic fiducial (Left) and two cubes (Upper right) may provide longer physical line angles (Lower right) to match with the virtual axe lines.

Most of the discrepancy between the virtual and the physical observed objects in this study was measured from the sagittal plane. Strateman et al. ${ }^{20}$ found significantly more measurement errors in the sagittal plane in the NewTom CBCT machine while Hassan et al. ${ }^{21}$ discovered that it may be less likely to detect root fracture of teeth in sagittal plane. However, in our previous study using the same iCAT CBCT machine, we did not find any trend of inaccuracy in specific plane of the $\mathrm{CBCT}^{10}$.

Registration of the unified coordination system in both the physical object and virtual imaging allows a bridging between two domains that may have many potential applications. In this paper, a transfer of the virtual planning to the reality with the use of static stent and simple surgical equipment was described. Moreover for real time surgical navigation/robotic surgery, unified coordination facilitates intra-operative registration and tracking of the RF corner and/or facial landmarks on the patient with the surgical handpiece in the surgeon's hand. This may extend to the field of the augmented reality, in which a head mounted display (HMD) or even a tablet/smartphone may show internal structures in the CBCT imaging after recognizing the surface coordination of a patient. A diagnosis of disease and surgical procedures would be assisted by seeing the imaging simultaneously. Furthermore, RF provides a directional information in the imaging particularly helpful in the interpretation of a 3D imaging with a limited field of view (FOV) for a reduction of radiation dosage. This would be important in obtaining a correct cross-sectional views for the treatment planning. The RF also helps an easy monitoring of serial imagings by incorporating a reference point, for which can be assessed easily both virtually and clinically. Lastly, in the part II of this paper, the accuracy of various imaging-imaging (II) registration algorithms (e.g. surface based, point based and pixel based) applied in different clinical situations may be compared and validated.

Registration of coordination do not limited to $\mathrm{CBCT}$ and may apply to other computerized virtual domains like 3D surface scan. By scanning the neck of placed implant ( $+/$ - scanning body for easier capture) and the corner of cube (fiducial), the implant apex can be estimated by knowing the implant length. Therefore, clinically the patient do not need to take postoperative CBCT to know the implant position and further reduce radiation suffered. 
Deviation of the transfer of surgical planning in this study was found to within the clinical safety margin of $2 \mathrm{~mm}^{22}$, however the discrepancy in relating the imaging to the reality must be considered when assess the clinical feasibility of particular computer guided implant surgery.

\section{CONCLUSION}

This paper presents a rigid registration in cone beam computed tomogram with acceptable clinical accuracy for mapping the virtuality to the reality, for measuring serial imagings, for potential use in navigation surgery and augmented reality. The mathematical model of the image calibration and registration (i.e. the mapping) has been described. A real case of a dental patient with missing maxillary (upper) left central incisor was collected and demonstrated for the proposed method in the image calibration and registration.

\section{REFERENCES}

[1] Lagravère, M. O., Carey, J, Toogood, R. W., Major, P. W., "Three-dimensional Accuracy of Measurements Made with Software on Cone-beam Computed Tomography Images,” Am. J. Orthod. Dentofacial Orthop 134(1), 112-116 (2008)

[2] Lascala, C. A., Panella, J., Marques, M. M., "Analysis of the Accuracy of Linear Measurements obtained by cone beam computed tomography (CBCT-NewTom)," Dentomaxillofac Radiol 33(5) 291-294 (2004)

[3] Wat, P. Y., Chow, T. W., Luk, H. W., Comfort, M. B., "Precision surgical template for implant placement: a new systematic approach,” Clin Implant Dent Relat Res 4(2) 88-92 (2002)

[4] Widmann, G., Bale, R. J., “Accuracy in computer-aided implant surgery--a review,” Int J Oral Maxillofac Implants 21(2) 305-13 (2006)

[5] Ganz, S. D., "Computer-aided design/computer-aided manufacturing applications using CT and cone beam CT scanning technology,” Dent Clin North Am 52(4) 777-808 (2008)

[6] Fadero, P. E., Shah, M., "Three dimensional (3D) modelling and surgical planning in trauma and orthopaedics ," Surgeon 12(6) 328-333 (2014)

[7] Wanschitz, F., Birkfellner, W., Watzinger, F., Schopper, C., Patruta, S., Kainberger, F., Figl, M., Kettenbach, J., Bergmann, H., Ewers, R., "Evaluation of accuracy of computer-aided intraoperative positioning of endosseous oral implants in the edentulous mandible," Clin Oral Implants Res 13(1) 59-64 (2002)

[8] Casap, N., Wexler, A., Persky, N., Schneider, A., Lustmann, J., "Navigation surgery for dental implants: assessment of accuracy of the image guided implantology system," J Oral Maxillofac Surg 62(9 Suppl 2) 116-9 (2004)

[9] Birkfellner, W., Figl, M., Huber, K., Watzinger, F., Wanschitz, F., Hummel, J., Hanel, R., Greimel, W., Homolka, P., Ewers, R., Bergmann, H., "A head-mounted operating binocular for augmented reality visualization in medicine-design and initial evaluation,” IEEE Trans Med Imaging 21(8) 991-7 (2002)

[10] Lam, W.Y.H., Ngan, H.Y.T., Wat, P.Y.P., Luk, H.W.K., Pow, E.H.N., Goto. T.K., "Novel geometric coordination in cone beam computed tomogram," Proc. IEEE AIPR Workshop, 140701-9 (2014)

[11] Besimo. C.E., Lambrecht, J.T., Guindy, J.S., “Accuracy of implant treatment planning utilizing template-guided reformatted computed tomography," Dentomaxillofac Radiol 29(1) 46-51 (2000)

[12] Ozan, O., Turkyilmaz, I., Ersoy, A.E., McGlumphy, E.A., Rosenstiel, S.F., "Clinical accuracy of 3 different types of computed tomography-derived stereolithographic surgical guides in implant placement," J Oral Maxillofac Surg 67(2) 394-401 (2009)

[13] Watson, T. F., Cook, R. J., "The influence of bur blade concentricity on high-speed tooth-cutting interactions: a videorate confocal microscopic study," J Dent Res 74(11) 1749-55 (1995)

[14] Watson, T.F., Flanagan, D., Stone, D.G., "High and low torque handpieces: cutting dynamics, enamel cracking and tooth temperature," Br Dent J. 24;188(12) 680-6 (2000)

[15] Pinsky, H. M., Dyda, S., Pinsky, R. W., Misch, K. A., Sarment, D. P., “Accuracy of three-dimensional measurements using cone-beam CT," Dentomaxillofac Radiol 35(6) 410-6 (2006) 
[16] Maurer, C. R. Jr., Fitzpatrick, J. M., Wang, M. Y., Galloway, R. L. Jr., Maciunas, R. J., Allen, G. S., "Registration of head volume images using implantable fiducial markers," IEEE Trans Med Imaging 16(4) 447-62 (1997)

[17] Fitzpatrick, J. M., West, J. B., Maurer, C. R. Jr., "Predicting error in rigid-body point-based registration," IEEE Trans Med Imaging 17(5) 694-702 (1998)

[18] Fitzpatrick, J. M., West, J. B.," The distribution of target registration error in rigid-body point-based registration," IEEE Trans Med Imaging 20(9) 917-27 (2001)

[19] Birkfellner, W., Solar, P., Gahleitner, A., Huber, K., Kainberger, F., Kettenbach, J., Homolka, P., Diemling, M., Watzek, G., Bergmann, H., "In-vitro assessment of a registration protocol for image guided implant dentistry," Clin Oral Implants Res 12(1) 69-78 (2001)

[20] Stratemann, S. A., Huang, J. C., Maki, K., Miller, A. J., Hatcher, D.C., "Comparison of cone beam computed tomography imaging with physical measures," Dentomaxillofac Radiol 37(2) 80-93 (2008)

[21] Hassan, B., Metska, M. E., Ozok, A. R., van der Stelt, P., Wesselink. P. R., "Comparison of five cone beam computed tomography systems for the detection of vertical root fractures," J Endod 36(1) 126-9 (2010)

[22] Greenstein, G., Tarnow, D., "The mental foramen and nerve: clinical and anatomical factors related to dental implant placement: a literature review," J Periodontol 77(12) 1933-43 (2006) 